\title{
The Chemical Enrichment and Mass Assembly Histories of SDSS Galaxies
}

\author{
R. Cid Fernandes ${ }^{1}$, N. V. Asari ${ }^{1}$, J. P. Torres-Papaqui ${ }^{1}$, \\ W. Schoenell ${ }^{1}$ L. Sodré Jr. ${ }^{2}$, A. Mateus ${ }^{2}$, and G. Stasińska ${ }^{3}$ \\ ${ }^{1}$ Universidade Federal de Santa Catarina, Florianópolis, SC, Brazil \\ ${ }^{2}$ Universidade de São Paulo, São Paulo, SP, Brazil \\ ${ }^{3}$ LUTH, Observatoire de Meudon, France
}

\begin{abstract}
We explore the mass-assembly and chemical enrichment histories of star forming galaxies by applying a population synthesis method to a sample of nearly $70 \mathrm{k}$ galaxies culled from over 500k galaxies from the Sloan Digital Sky Survey Data Release 5. Our method decomposes the entire observed spectrum in terms of a sum of simple stellar populations spanning a wide range of ages and metallicities, thus allowing the reconstruction of galaxy histories. A comparative study of galaxy evolution is presented, where galaxies are grouped onto bins of nebular abundances or mass. We find that galaxies whose warm interstellar medium is poor in heavy elements are slow in forming stars. Their stellar metallicities also rise slowly with time, reaching their current values $\left(Z_{\star} \sim 1 / 4 Z_{\odot}\right)$ in the last $\sim 100 \mathrm{Myr}$ of evolution. Systems with metal rich nebulae, on the other hand, assembled most of their mass and completed their chemical evolution long ago, reaching $Z_{\star} \sim Z_{\odot}$ already at lookback times of a few Gyr. These same trends, which are ultimately a consequence of galaxy downsizing, appear when galaxies are grouped according to their stellar mass. The reconstruction of galaxy histories to this level of detail out of integrated spectra offers promising prospects in the field of galaxy evolution theories.
\end{abstract}

Keywords. galaxies: evolution - galaxies: stellar content - galaxies: statistics

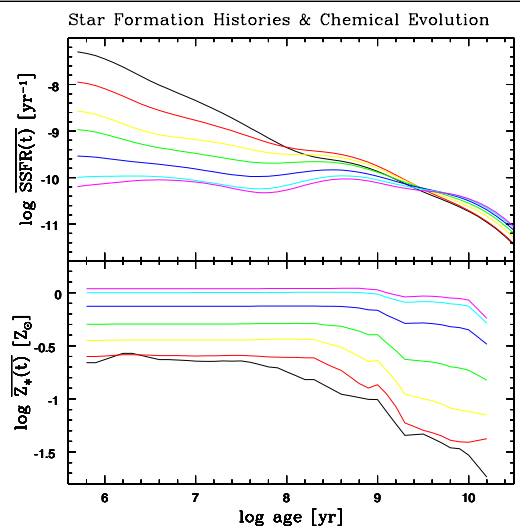

Figure 1. Top: Average Specific Star Formation Rate as a function of lookback time for galaxies in six different bins of present day oxygen abundance (measured from emission lines). Lower $\mathrm{O} / \mathrm{H}$ bins are those with higher SSFR in the recent past. The lower $\mathrm{O} / \mathrm{H}$ (or, equivalently, the lower the mass of a galaxy), the higher is its recent SSFR with respect to the past. Bottom: Evolution of the mean stellar metallicity for galaxies in the same six bins in $\mathrm{O} / \mathrm{H}$. Galaxies with low $\mathrm{O} / \mathrm{H}$ are notably slow in their chemical evolution compared to massive, large $\mathrm{O} / \mathrm{H}$ galaxies, which have completed their evolution long ago. 